\title{
Lateral Ventricle
}

National Cancer Institute

\section{Source}

National Cancer Institute. Lateral Ventricle. NCI Thesaurus. Code C12834.

The rostral extensions of the ventricular system of the brain consisting of two cavities, one on each side of the brain within the central regions of each cerebral hemisphere.

Cerebrospinal fluid flows from the lateral ventricles into the centrally third ventricle via the foramen of Monroe. 